\title{
FINITE GROUPS AS GALOIS GROUPS OF FUNCTION FIELDS WITH INFINITE FIELD OF CONSTANTS
}

\author{
C. ÁLVAREZ-GARCÍA and G. VILLA-SALVADOR ${ }^{凶}$
}

(Received 5 June 2009; accepted 15 January 2010)

Communicated by F. Calegari

\begin{abstract}
Let $E / k$ be a function field over an infinite field of constants. Assume that $E / k(x)$ is a separable extension of degree greater than one such that there exists a place of degree one of $k(x)$ ramified in $E$. Let $K / k$ be a function field. We prove that there exist infinitely many nonisomorphic separable extensions $L / K$ such that $[L: K]=[E: k(x)]$ and $\operatorname{Aut}_{k} L=\operatorname{Aut}_{K} L \cong \operatorname{Aut}_{k(x)} E$.

2000 Mathematics subject classification: primary 12F12; secondary 11R58, 12F10, $14 \mathrm{H} 05$.

Keywords and phrases: automorphism groups, function fields, infinite field of constants, nonseparably generated function fields.
\end{abstract}

\section{Introduction}

Let $K$ be an algebraic function field over a field $k$ and let Aut $_{k} K$ be its full group of automorphisms. For $k=\mathbb{C}$, Greenberg [4] proved in 1974 that given a nontrivial finite group $G$, there exist infinitely many Galois extensions $L / K$ such that $\operatorname{Gal}(L / K)$ is isomorphic to $G$ and that $\operatorname{Gal}(L / K)=\operatorname{Aut}_{\mathbb{C}} L$. This result of Greenberg gives a positive answer to the inverse problem in Galois theory for function fields $K / \mathbb{C}$.

In several other cases Greenberg's result can be extended to function fields $K / k$ with $k$ an algebraically closed field.

Madden and Valentini [6] proved that every finite group can be realized as the full automorphism group of an algebraic function field $K / k$. D'Mello and Madan [3] established the theorem of Greenberg in the case where $G$ is a solvable group $G$ and $K=k(x)$ is a rational function field.

The main result of Stichtenoth in [8] is that if $E / k(x)$ a finite separable extension of degree greater than one, then, for every function field $K / k$ of genus greater than one, there exist infinitely many nonisomorphic separable extensions $L / K$ such that

$$
[E: k(x)]=[L: K] \text { and } \operatorname{Aut}_{k(x)} E \cong \operatorname{Aut}_{K} L=\operatorname{Aut}_{k} L .
$$

(C) 2010 Australian Mathematical Publishing Association Inc. 1446-7887/2010 \$16.00 
The results of Stichtenoth and of D'Mello and Madan can be combined to obtain the analogous of Greenberg's theorem provided that the group $G$ is solvable and the genus of $K$ is larger than one.

Madan and Rosen [5] proved that Greenberg's result remains valid for an arbitrary function field $K / k$ with $k$ an algebraically closed field of arbitrary characteristic. This result gives a positive answer to the inverse problem of Galois theory for any field $K$ over an algebraically closed field of constants.

Rzedowski and Villa [7] proved an analogue of Stichtenoth's result for congruence function fields without restriction on the genus, provided that in the extension $E / k(x)$ there exist prime divisors of degree one, one ramified and another unramified. In [1] we remove the ramification restrictions given in [7].

A natural question is what happens when the field of constants $k$ of a function field is an arbitrary field. Some of the results are straightforward for separably generated function field extensions $K / k$, for instance, when $k$ is a perfect field. If $K / k$ is not a separably generated extension, some of the tools we have in the case where $k$ is perfect are no longer available; for instance the Castelnuovo-Severi inequality, the difference of the extension, and so on. We also have to deal with the special behavior of new constants and so on.

The main goal of this paper is to establish an analogue of the main result of [7] for an infinite field of constants $k$ now under one ramification restriction. We prove that given any infinite field $k$, if $E / k(x)$ is any separable extension, where $k$ is the full field of constants of $E$, such that a place of $k(x)$ of degree one is ramified in $E$, then for any function field $K / k$, there exist infinitely many nonisomorphic function fields over $k$ such that $L / K$ is a separable extension of the same degree as $E / k(x)$ and $\operatorname{Aut}_{K} L=\operatorname{Aut}_{k} L \cong \operatorname{Aut}_{k(x)} E$. This is Theorem 4.3.

Given a function field $K / k$, we first choose a suitable $y \in K$ and construct a suitable $C$-improvement $E_{1} / k(y)$ of $E / k(x)$ (see [7]) such that the field of constants of $L=E_{1} K$ is $k$, while [ $\left.L: K\right]=[K: k(y)]$ and the intermediate fields of $L$ other than $K$ have large enough genus (Proposition 3.5). With this condition, it follows that any element of the group of automorphisms of $L$ over $k$ restricts to an automorphism of $K$ (Proposition 3.6). The next step is to find a ramification of the places in the support of $(y)_{K}$ in such a way that any automorphism of $L$ over $k$ fixes $K$ elementwise. If the constant field is finite or algebraically closed then the extension $K / k(y)$ is separable. We use the $C$-improvements constructed in Proposition 4.1 to deal with the inseparable case.

The paper is organized as follows. In Section 2 we give several general results that will be used to find field extensions with suitable properties to be used in our construction. In Section 3 we find a bound for the genus of the compositum of fields. When $K / k$ is separably generated, we have the Castelnuovo-Severi inequality. We find an analogue of this bound of the genus for general $k$. This is Proposition 3.5. As a consequence of this variant of the Castelnuovo-Severi inequality, we have the analogue of a result of Madden and Valentini [6] that establishes that if $L / K$ is an extension such that every proper intermediate field has large enough genus, then for any automorphism $\sigma$ of $L$, we have $\sigma(K)=K$. 
Section 4 deals with function field extensions with a prime divisor of degree one ramified. Under this ramification restriction it is possible to prove our main result.

We use the following notation. The field $k$ is an infinite field and $K / k$ denotes a function field with full field of constants $k$. In a rational function field $k(x)$, $P_{f}$ denotes the place defined by the irreducible polynomial $f$. For a field extension $F / K$, the group of automorphisms of $L$ that fix $K$ pointwise is denoted by Aut $_{K} F$. For a function field $E / k$, let $(x)_{E}$ denote the principal divisor of $x$ in $E$ and $\operatorname{deg} P$ denote the degree of a place $P$ of $E$. For an extension $F / E, \operatorname{Con}_{F / E}$ is the conorm map with respect to $F / E$. If $F / E$ is a separable extension we write $D_{F / E}$ for the different of the extension.

\section{Function fields over infinite field of constants}

In this section we give some general results that will be needed to prove the main result of the paper.

LEMMA 2.1. Let $F / k(x)$ be a Galois extension and let $k(y)$ be a rational function field such that $([F: k(x)],[k(y): k(x)])=1$. If $E / l$ is an intermediate field of $F / k(x)$, then the field of constants of $E(y)$ is $l$.

PROOF. Let $N$ be the field of constants of $E(y)$. Since $N / l$ is a separable we have that $[E: l(x)]=[E N: N(x)]$. Since $[k(y): k(x)]=[N(y): N(x)]$, it follows that $([E N: N(x)],[N(y): N(x)])=1$. Hence $[E(y): N(y)]=[E N: N(x)]$.
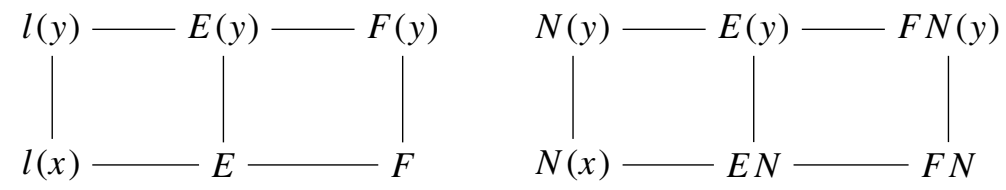

Similarly $[E(y): l(y)]=[E: l(x)]$, and so we obtain $[E(y): N(y)]=[E(y): l(y)]$. Therefore $N(y)=l(y)$ and $N=l$.

The proof of Lemma 2.2 is similar to the proof in the case where the constant field is perfect [9].

LEMMA 2.2. Suppose that $F^{\prime} / F$ is a finite separable extension of function fields. Let $F_{1}, F_{2}$ be intermediate fields of $F^{\prime} / F$ such that $F^{\prime}=F_{1} F_{2}$. Then, for a place $P \in \mathbb{P}_{F}$ :

(1) if $P$ is completely decomposed in $F_{1} / F$ and $F_{2} / F$, then $P$ is completely decomposed in $F^{\prime} / F$;

(2) if $P$ is unramified and separable in $F_{1} / F$ and $F_{2} / F$, then $P$ is unramified and separable in $F^{\prime} / F$.

As a consequence of (2) of Lemma 2.2 we have the following lemma.

LEMMA 2.3. Let $K / k$ be a function field and let $E / K$ be a finite separable extension with normal closure $\tilde{E}$. Assume that $P \in \mathbb{P}_{K}$ is ramified or inseparable in $\tilde{E}$. Then $P$ is ramified or inseparable in $E$. 
Lemma 2.4. Suppose that $E / K$ is a finite separable extension of function fields. Let $P$ be a place of $K$ ramified or inseparable in $E$. Then for a purely inseparable finite extension $F / K$ the place $B$ of $F$ lying over $P$ is ramified or inseparable in $E F$.

PROOF. Let $\tilde{E}$ be the normal closure of $E / K$. Since $\tilde{E} \cap F=K$, the normal closure of $E F / F$ is $\tilde{E} F$. Now we consider a place $P_{1}$ of $\tilde{E}$ lying over $P$ and let $B_{1}$ be the extension of $P_{1}$ in $\tilde{E} F$. Since $B_{1} \cap F$ is over $P$ we have that $B_{1} \cap F=B$.

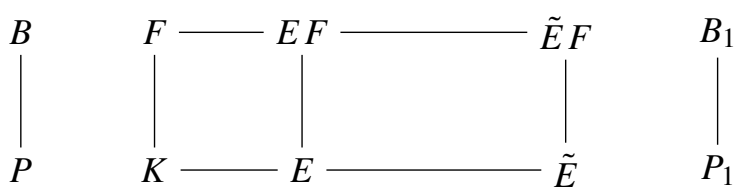

Denote by $D\left(P_{1} \mid P\right)$ the decomposition group of $P_{1}$ and by $I\left(P_{1} \mid P\right)$ the inertia group. The restriction of an element in $D\left(B_{1} \mid B\right)$ to $\tilde{E}$ belongs to $D\left(P_{1} \mid P\right)$, so there is an embedding $\varphi: D\left(B_{1} \mid B\right) \rightarrow D\left(P_{1} \mid P\right)$ such that $\varphi\left(I\left(B_{1} \mid B\right)\right) \subseteq I\left(P_{1} \mid P\right)$. Since the place $B_{1}$ is the only extension of $P_{1}$ in $\tilde{E} F, \varphi$ is an isomorphism.

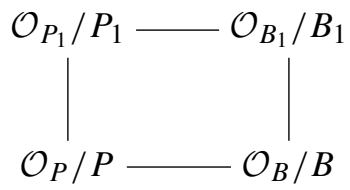

On the other hand, $\left[\mathcal{O}_{B_{1}} / B_{1}: \mathcal{O}_{B} / B\right]_{s}=\left[\mathcal{O}_{P_{1}} / P_{1}: \mathcal{O}_{P} / P\right]_{s}$ since the extensions $\left(\mathcal{O}_{B} / B\right) /\left(\mathcal{O}_{P} / P\right),\left(\mathcal{O}_{B_{1}} / B_{1}\right) /\left(\mathcal{O}_{P_{1}} / P_{1}\right)$ are purely inseparable. Hence

$$
\begin{aligned}
{\left[D\left(P_{1} \mid P\right): I\left(P_{1} \mid P\right)\right] } & =\left[\mathcal{O}_{P_{1}} / P_{1}: \mathcal{O}_{P} / P\right]_{s}=\left[\mathcal{O}_{B_{1}} / B_{1}: \mathcal{O}_{B} / B\right]_{s} \\
& =\left[D\left(B_{1} \mid B\right): I\left(B_{1} \mid B\right)\right] .
\end{aligned}
$$

Therefore $\varphi\left(I\left(B_{1} \mid B\right)\right)=I\left(P_{1} \mid P\right)$, so $B$ is ramified or inseparable in $\tilde{E} F / F$. Finally, from Lemma 2.3 we obtain that $B$ is ramified or inseparable in $E F / F$.

LEMMA 2.5. Let $E / K$ be a Galois extension of algebraic functions fields with field of constants $k$. Let $\sigma \in \operatorname{Gal}(E / K)$. Then if $\sigma \neq \operatorname{Id}$ the set $A_{\sigma}=\left\{B \in \mathbb{P}_{E} \mid \sigma(B) \neq B\right\}$ is infinite.

Proof. Suppose that $A_{\sigma}$ is finite for some $\sigma \neq \mathrm{Id}$. Let $K_{1}$ be the fixed field of $\sigma$ and $A_{\sigma}=\left\{B_{1}, \ldots, B_{m}\right\}$. Now $\sigma(B)=B$ for each place $B \in \mathbb{P}_{E}$ with $B \neq B_{i}$. Let $y_{1} \in E \backslash K_{1}$ and $y_{2} \in K_{1}$ be such that $v_{B_{i}}\left(y_{2}\right)>0$. Then there exists $j$ such that $v_{B_{i}}\left(y_{1} y_{2}^{j}\right)>0$ and $y_{1} y_{2}^{j} \notin K_{1}$. Let $c$ be a constant distinct from 1 and let $y=y_{1} y_{2}^{j}+c$, $y \notin K_{1}$. Hence $v_{B_{i}}(y)=v_{B_{i}}(y+1)=0$. It follows that $\sigma(y)=y$, which implies that $\sigma(y)=a y$ with $a \in k$. Similarly, there exists $b \in k$ such that $\sigma(1+y)=b(1+y)$, then $1+a y=b+b y$. Since $y \notin k$ we obtain that $\sigma(y)=y$. Thus $y \in K_{1}$. This contradiction shows the result. 


\section{Bound for the genus of a compositum of fields}

The Castelnuovo-Severi inequality is valid for separably generated extensions $K / k$. This inequality plays an important role in [7, 8]. In Proposition 3.5 we give an analogous inequality for $K / k$ not necessarily separable generated. Then we deduce Proposition 3.6 which is the main result of this section. This is the analogue of the Madden-Valentini result in [6].

For a function field $K / k$ of genus $g_{K}$, let $g_{K}^{\prime}=\max \left\{g_{K}, 1\right\}$.

LEMMA 3.1. Let $F / k$ be a function field with separably closed field of constants. Given a separable geometric finite extension $E / F$, there exist infinitely many places in $F$ that are completely decomposed in $E / F$. If $d=\min \left\{\operatorname{deg} P \mid P \in \mathbb{P}_{F}\right\}$ we can choose the places of degree less than or equal to $2 g_{F}^{\prime} d$.

PRoOf. Let $P$ be a place of $F$ such that $d=\operatorname{deg} P$. There exists $x \in F$ with pole divisor $P^{2 g_{F}^{\prime}}$. Denote by $F_{0}$ the separable closure of $k(x)$ in $F$ and by $E_{0}$ the separable closure of $k(x)$ in $E$. Since $E_{0}$ and $F$ are linearly disjoint over $F_{0}$ and $[E: k(x)]_{i}=[F: k(x)]_{i}$, then $E=E_{0} F$.

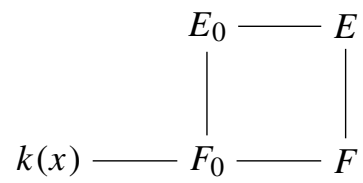

In the extension $E_{0} / k(x)$ there are a finite number of places which are either ramified or inseparable. Hence almost all places of degree one of $k(x)$ are unramified and separable in $E_{0} / k(x)$, so these places are completely decomposed in $E_{0} / k(x)$. Therefore there exist infinitely many places of degree one in $F_{0}$ which are completely decomposed in $E_{0}$.

Let $Q$ be a place of degree one in $F_{0}$ completely decomposed in $E_{0}$ and let $R$ be the place of $F$ lying over $Q$. Let $\tilde{E}_{0}$ be a normal closure of $E_{0} / F_{0}$. Lemma 2.2 implies that $Q$ is completely decomposed in $\tilde{E}_{0} / F_{0}$.
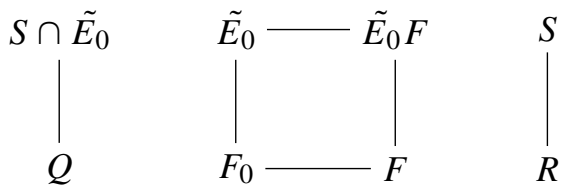

Since, given a place $S$ of $\tilde{E}_{0} F$ lying over $R$, the decomposition group $D(S \mid R)$ embeds in the group $D\left(S \cap \tilde{E}_{0} \mid Q\right)$, then $R$ is completely decomposed in $E_{0} F / F$. The relative degree $f(R \mid Q)=\operatorname{deg} R$ is less than or equal to $[F: k(x)]=2 g_{F}^{\prime} d$.

LEMMA 3.2. Assume that $k$ is separably closed, and consider a function field $F / k$ such that $F_{1} / k$ is a subfield of $F$ such that $F / F_{1}$ is a separable extension of degree $n>1$. Let $y \in F$ be an element such that $F=F_{1}(y)$ and $d_{1}=\min \left\{\operatorname{deg} P \mid P \in \mathbb{P}_{F_{1}}\right\}$. 
Then there exist infinitely many places $P \in \mathbb{P}_{F_{1}}$ of degree at most $2 g_{F_{1}}^{\prime} d_{1}$ having the following properties.

(1) $P$ has $n$ distinct extensions $P_{1}, \ldots, P_{n}$ in $F / F_{1}$.

(2) The restrictions $P_{1} \cap k(y), \ldots, P_{n} \cap k(y)$ are pairwise distinct places of $k(y)$.

PROOF. Let $\varphi(z)=z^{n}+a_{n-1} z^{n-1}+\cdots+z_{0} \in F_{1}[z]$ be the minimal polynomial of $y$ over $F_{1}$. By Lemma 3.1 there are infinitely many places $P \in \mathbb{P}_{F_{1}}$ completely decomposed in $F / F_{1}$ and such that $\left\{1, \ldots, y^{n-1}\right\}$ is an integral basis of $F / F_{1}$ for almost all such places $P$. Since by Kummer's theorem the decomposition of the polynomial $\bar{\varphi}(z) \in\left(\mathcal{O}_{P} / P\right)[z]$ corresponds to the decomposition of $P$ in $F$, we must have that $\bar{\varphi}(z)=\prod_{i=1}^{n}\left(z-c_{i}\right)$ with pairwise distinct elements $c_{i} \in \mathcal{O}_{P} / P$.

Let $b_{i}$ be such that $c_{i}=b_{i}+P$. Again by Kummer's theorem we have that for $i=1, \ldots, n$ there exists a unique place $P_{i} \in \mathbb{P}_{F}$ such that $P_{i} \mid P$ and $v_{P_{i}}\left(y-b_{i}\right)>0$. There exist $\beta_{i} \in k$ and an integer $m \geq 0$ such that $b_{i}^{p^{m}}+P=\beta_{i}+P$. Hence

$$
v_{P_{i}}\left(y^{p^{m}}-\beta_{i}\right) \geq \min \left\{v_{P_{i}}\left(y^{p^{m}}-b_{i}^{p^{m}}\right), v_{P_{i}}\left(b_{i}^{p^{m}}-\beta_{i}\right)\right\}>0 .
$$

Since $\beta_{i}=\beta_{j}$ implies $c_{i}^{p^{m}}=c_{j}^{p^{m}}$, it follows that the elements $\beta_{i}$ are pairwise distinct, so the restrictions $P_{i} \cap k\left(y^{p^{m}}\right)$ are distinct.

The following result can be found in [9].

Lemma 3.3. Let $F_{1} / k$ be a subfield of $F / k$ and let $\left[F: F_{1}\right]=n$. Assume that $\left\{z_{1}, \ldots, z_{n}\right\}$ is a basis of $F / F_{1}$ such that all $z_{i} \in L\left(C^{-1}\right)$ for some divisor $C \in D_{F}$. Then $g_{F} \leq 1+n\left(g_{F_{1}}-1\right)+\operatorname{deg} C$.

LEMMA 3.4. Let $F / k$ be a function field with separably closed field of constants. Suppose that $F_{1} / k$ and $F_{2} / k$ are subfields of $F / k$ satisfying the following conditions.

(1) $F=F_{1} F_{2}$ and $F / F_{1}$ is separable.

(2) $\quad\left[F: F_{i}\right]=n_{i}$ and $F_{i} / k$ has genus $g_{i}(i=1,2)$.

Then the genus $g$ of $F / k$ is bounded above by $1+n_{1}\left(g_{1}-1\right)+4 n_{1} n_{2} g_{1}^{\prime} g_{2}^{\prime} d_{1}$, where $d_{1}=\min \left\{\operatorname{deg} P \mid P \in \mathbb{P}_{F_{1}}\right\}$.

Proof. Since $F=F_{1} F_{2}$ there are $y_{1}, \ldots, y_{s} \in F_{2}$ with $F=F_{1}\left(y_{1}, \ldots, y_{s}\right)$. The extension $F / F_{1}$ is separable, hence we can find $a_{1}, \ldots, a_{s} \in k$ such that the element $y=\sum a_{j} y_{j} \in F_{2}$ is a primitive element of $F / F_{1}$. Let $P \in \mathbb{P}_{F_{1}}$ be such that it has $n_{1}$ distinct extensions $P_{1}, \ldots, P_{n_{1}}$ in $F$ and such that the restrictions $Q_{i}=P_{i} \cap F_{2} \in \mathbb{P}_{F_{2}}$ are pairwise distinct (Lemma 3.1). We have that deg $Q_{i}=2 g_{1}^{\prime} d_{1}$. There exists $u_{i} \in F_{2}$ with pole divisor $Q_{i}^{2 g_{2}^{\prime}}$. The elements $u_{1}, \ldots, u_{n_{1}}$ form a basis of $F / F_{1}$.

Suppose that $\sum x_{i} u_{i}=0$ with $x_{i} \in F_{1}$ is a nontrivial linear combination. Let $j \in\left\{1, \ldots, n_{1}\right\}$ be such that $v_{P}\left(x_{j}\right) \leq v_{P}\left(x_{i}\right)$ for $i=1, \ldots, n_{1}$. Then

$$
v_{P_{j}}\left(x_{j} u_{j}\right)=v_{P_{j}}\left(x_{j}\right)+v_{P_{j}}\left(u_{j}\right) \leq v_{P}\left(x_{j}\right)-2 g_{2}^{\prime} .
$$


For $i \neq j$,

$$
v_{P_{j}}\left(x_{i} u_{i}\right)=v_{P_{j}}\left(x_{i}\right)+v_{P_{j}}\left(u_{i}\right) \geq v_{P}\left(x_{i}\right),
$$

hence $v_{P_{j}}\left(\sum x_{i} u_{i}\right)=v_{P_{j}}\left(x_{j} u_{j}\right)<\infty$, which is not possible. Now we consider the divisor $C=\operatorname{Con}_{F / F_{2}}\left(\sum Q_{i}^{2 g_{2}^{\prime}}\right)$. Its degree is $2 n_{2} g_{2}^{\prime} \sum \operatorname{deg} Q_{i} \leq 4 n_{1} n_{2} g_{1}^{\prime} g_{2}^{\prime} d_{1}$. Since the elements $u_{1}, \ldots, u_{n_{1}}$ are in $L\left(C^{-1}\right)$, we have $g \leq 1+n_{1}\left(g_{1}-1\right)+4 n_{1} n_{2} g_{1}^{\prime} g_{2}^{\prime} d_{1}$ from Lemma 3.3.

PROposition 3.5. Let $F, F_{1}, F_{2}$ be function fields with field of constants $k$ such that $F=F_{1} F_{2}$ and $F / F_{1}$ is a separable extension. Then

$$
g_{F} \leq 1+\left[F: F_{1}\right]\left(g_{F_{1}}-1\right)+4\left[F: F_{1}\right]\left[F: F_{2}\right] g_{F_{1}}^{\prime} g_{F_{2}}^{\prime} d,
$$

where $d=\min \left\{\operatorname{deg} P \mid P \in \mathbb{P}_{F_{1}}\right\}$.

Proof. Consider a separable closure $k_{1}$ of $k$ and let $\tilde{F}=F k_{1}, \tilde{F}_{i}=F_{i} k_{1}, i=1,2$. Since $k_{1} / k$ is separable, $g_{\tilde{F}}=g_{F}, g_{\tilde{F}_{i}}=g_{F_{i}}$ and $n_{i}=\left[\tilde{F}: \tilde{F}_{i}\right]=\left[F: F_{i}\right], i=1,2$. Let $d_{1}=\min \left\{\operatorname{deg} R \mid R \in \mathbb{P}_{\tilde{F}_{1}}\right\}$. By Lemma 3.4,

$$
\begin{aligned}
g_{F}=g_{\tilde{F}} & \leq 1+n_{1}\left(g_{\tilde{F}_{1}}-1\right)+4 n_{1} n_{2} g_{\tilde{F}_{1}}^{\prime} g_{\tilde{F}_{2}}^{\prime} d_{1} \\
& =1+\left[F: F_{1}\right]\left(g_{F_{1}}-1\right)+4\left[F: F_{1}\right]\left[F: F_{2}\right] g_{F_{1}}^{\prime} g_{F_{2}}^{\prime} d_{1} .
\end{aligned}
$$

We choose $P \in \mathbb{P}_{F_{1}}$ such that $\operatorname{deg} P=d$, and let $R$ be a place in $\tilde{F}_{1}$ lying over $P$. Now $d=\left[\mathcal{O}_{P} / P: k\right] \geq\left[\mathcal{O}_{R} / R: k_{1}\right] \geq d_{1}$ since $\mathcal{O}_{R} / R$ is the composition of $\mathcal{O}_{P} / P$ and $k_{1}$ [2, p. 128], and the result follows.

The following result is a consequence of Proposition 3.5 with $F_{1}=K, F_{2}=\sigma(K)$.

PROPOSITION 3.6. If $L / K$ is a finite separable extension of function fields with field of constants $k$ such that, for each intermediate field $M, K \subset M \subseteq L$,

$$
g_{M}>1+[M: K]\left(g_{K}-1\right)+4[M: K]^{2} g_{K}^{\prime 2} d,
$$

then for each $\sigma \in \mathrm{Aut}_{k} L$ we obtain that $\sigma(K)=K$.

\section{Separable extensions with a prime divisor of degree one ramified}

The next result is analogous to [8, Lemma 2] and [7, Lemma 2] and we will use it to find suitable $C$-improvements of a given finite separable extension $E / k(x)$.

Proposition 4.1. Assume that $k$ is infinite and let $E / k(x)$ be a finite separable extension of function fields over $k$ such that $P_{x-1}$ is ramified in $E$ and the zero $Q_{1}$ and the pole $Q_{2}$ of $x$ in $k(x)$ are unramified and separable in $E$. Let $\tilde{E} / l$ be a normal closure of $E / k(x)$. Let $C, C_{1}, C_{2} \in \mathbb{R}^{+}$be arbitrary. Then there exists a finite extension $F / k(y)$ satisfying the following properties. 
(1) There exists a subfield $E_{1} / k$ of $F / l$ such that

$$
\begin{gathered}
{[E: k(x)]=\left[E_{1}: k(y)\right], \quad[\tilde{E}: k(x)]=[F: k(y)],} \\
\operatorname{Gal}(E / k(x)) \cong \operatorname{Gal}\left(E_{1} / k(y)\right)
\end{gathered}
$$

and $F$ is a normal closure of $E_{1} / k(y)$. Moreover, $P_{y-1}$ is ramified in $F$ and the pole of $y$ in $k(y)$ is unramified and separable in $F$.

(2) Let $E_{2}$ be an intermediate field, $k(y) \subset E_{2} \subseteq E_{1}$. Then either there is a place in $k(y)$ of degree greater than $C_{2}$ ramified or inseparable in $E_{2} / k(y)$ or there are more than $C_{1}$ places of $k(y)$ ramified or inseparable in $E_{2} / k(y)$.

(3) For each intermediate field $k \subseteq k_{1} \subseteq l$, the genus $g_{M}$ of any field $k_{1}(y) \subset M \subseteq F$ with constant field $k_{1}$ is greater than $C$.

(4) Let $N / k$ be a finite separable extension. If $M / N$ is any intermediate field, $N(y) \subset M \subseteq F N$, then $g_{M}>C$.

Proof. First we prove (1) and (2). Choose an integer $m>0$ such that $m>$ $\max \left\{C_{1} C_{2}, 2 C+3\right\}$ and $(m, p[\tilde{E}: k(x)])=1$. Let $y^{m}=x$. Then $k(y) / k(x)$ is a separable extension of degree $m$. By the genus formula the only places of $k(x)$ which ramify in $k(y) / k(x)$ are $Q_{1}$ and $Q_{2}$, and there are no inseparable places. Let $F=\tilde{E}(y)$. Then $F$ is the normal closure of $E_{1}=E(y),\left[E_{1}: k(y)\right]=[E: k(x)]$, the constant field of $E_{1}$ is $k$ and $\operatorname{Aut}_{k(y)} E_{1} \cong \operatorname{Aut}_{k(x)} E$ (Lemma 2.1).

Consider an intermediate field $E_{0}, k(x) \subset E_{0} \subseteq E$. By the genus formula there is a place $Q_{f}$ of $k(x)$ ramified or inseparable in $E_{0}$. Let $\tilde{E}_{0}$ be the normal closure of $E_{0} / k(x)$ contained in $\tilde{E}$. Since $Q_{f}$ is different from $Q_{1}$ and $Q_{2}$ it follows that $Q_{f}$ is unramified in $k(y) / k(x)$; this implies that the polynomial $f(x)=f\left(y^{m}\right)$ splits into irreducible distinct factors $f_{1}(y), \ldots, f_{h}(y)$ in $k(y)$.

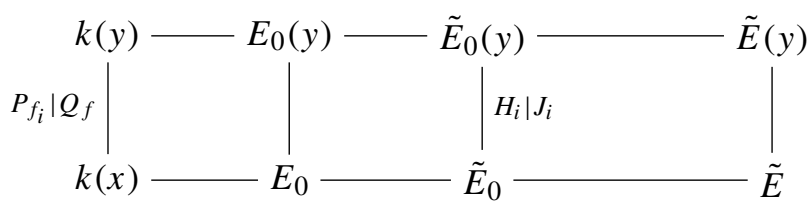

Let $H_{i}$ be an extension of $P_{f_{i}}$ in $\tilde{E}_{0}(y)$. Since $J_{i}=H_{i} \cap \tilde{E}_{0}$ lies over $Q_{f}$, then $J_{i}$ is ramified or inseparable in $\tilde{E}_{0} / k(x)$, so $e\left(H_{i} \mid Q_{i}\right) f_{i}\left(H_{i} \mid Q_{i}\right)>1$. Since $P_{f_{i}} \cap k(x)$ is different from $Q_{1}$ and $Q_{2}$, the place $P_{f_{i}}$ is unramified and separable in $k(y) / k(x)$, hence $e\left(H_{i} \mid P_{f_{i}}\right) f_{i}\left(H_{i} \mid P_{f_{i}}\right)>1$. Then $P_{f_{i}}$ is ramified or inseparable in $E_{0}(y) / k(y)$ (Lemma 2.3). Since $P_{y-1} \mid Q_{x-1}$, it follows that $P_{y-1}$ is ramified in $F$. Since $Q_{2}$ is unramified and separable in $\tilde{E} / k(x)$ (Lemma 2.2), the pole of $y$ is unramified and separable in $F / k(y)$ by the correspondence between the inertia groups. If $\operatorname{deg} f_{i} \leq C_{2}$ for all $i$, the number $h$ of factors is minimum when $\operatorname{deg} f_{i}=C_{2}$. In this case $h C_{2}=m \operatorname{deg} f>C_{1} C_{2}$, and it follows that $h>C_{1}$.

To prove (3), let $k_{1}$ be an intermediate field of $l / k$. Denote by $R_{1}$ the zero of $x$ in $k_{1}(x)$ and by $R_{2}$ the pole of $x$ in $k_{1}(x)$. The extension $k_{1}(x) / k(x)$ has degree equal 
to $f\left(R_{1} \mid Q_{1}\right)=f\left(R_{2} \mid Q_{2}\right)$. Therefore if a place $D_{i} \in \mathbb{P}_{k_{1}(y)}$ lies over $R_{i}$ we obtain $e\left(D_{i} \mid R_{i}\right)=[k(y): k(x)]=m$. Then in $k_{1}(y), R_{i}=D_{i}^{m}$ with $i=1,2$.
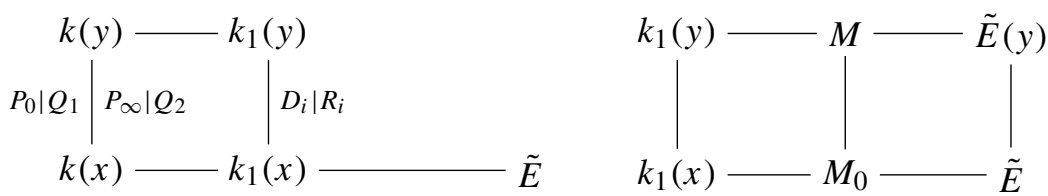

Let $M / k_{1}$ be such that $k_{1}(y) \subset M \subseteq \tilde{E}(y)$. If $M_{0}=M \cap \tilde{E}$, then $k_{1}(x) \subset M_{0} \subseteq \tilde{E}$. Let $T \in \mathbb{P}_{M}$ be such that $T \mid D_{i}$. Since $(m,[\tilde{E}: k(x)])=1$, then $e\left(T \mid T \cap M_{0}\right) \geq m$. Since $\left[M: M_{0}\right]=m$, it also follows that $e\left(T \mid T \cap M_{0}\right)=m$ and $f\left(T \mid T \cap M_{0}\right)=1$. Since $Q_{1}$ is unramified in $\tilde{E}$ we have that in $M_{0}, R_{1}=T_{1} \cdots T_{h}$, where $h \geq 2$ or $f\left(T_{1} \mid R_{1}\right) \geq 2$.
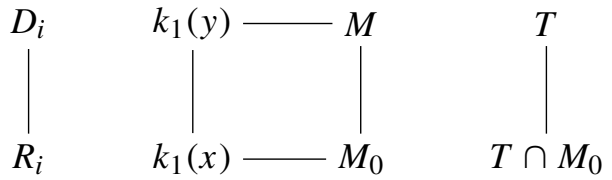

Then at least three places of $M$ are fully ramified in $M / M_{0}$ or at least two places are fully ramified and one of them is of degree greater than or equal to two. From the genus formula we obtain that

$$
\begin{aligned}
g_{M} & =1+m\left(g_{M_{0}}-1\right)+\frac{1}{2} \operatorname{deg}\left(D_{M / M_{0}}\right) \\
& \geq 1-m+\frac{3}{2}(m-1)=1+\frac{1}{2}(m-3) .
\end{aligned}
$$

Finally we prove (4). Let $k_{1}=N \cap l$. Then $k_{1}(y) \subseteq N(y) \cap F$. If $M / N$ is any intermediate field, $N(y) \subset M \subseteq F N$ and $M_{1}=M \cap F$, then $N(y) \cap F \subset M_{1} \subseteq F$.

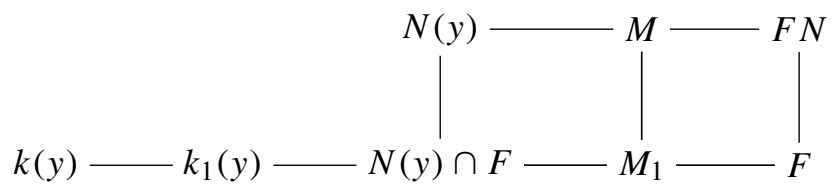

The constant field of $M_{1}$ is $k_{1}$ and $M=M_{1} N$. This implies that $g_{M}=g_{M_{1}}$, and by (3) it follows that $g_{M_{1}}>C$.

Proposition 4.2. Suppose that $K / k$ is a function field such that $K / k(y)$ is a finite extension, where $K_{0}$ is the separable closure of $k(y)$ in $K$. Let $C_{0} \in \mathbb{R}^{+}$and let $n_{s}$ be the number of places $P$ of $k(y)$ which are ramified or inseparable in $K_{0} / k(y)$. Assume that the degree of these places is less than $d_{s}$. Let $F / k(y)$ be an extension with the properties stated in Proposition 4.1 such that $C>g_{K_{0}}$ and $C_{1}>n_{s}+2\left(m+C_{0}\right)$, $C_{2}>d_{s}, 2\left(m+C_{0}\right)$, where $m=[E: k(x)]$. Then the following hold.

(1) The constant field of $E_{1} K$ is $k$. 
(2) $\left[E_{1}: k(y)\right]=\left[E_{1} K: K\right]$, whence $\operatorname{Aut}_{k(y)} E_{1} \cong$ Aut $_{K} E_{1} K$ and $F K$ is the normal closure of $E_{1} K$.

(3) Each field $H$ such that $K \subset H \subseteq E_{1} K$ has genus greater than $C_{0}$.

ProOF. First we prove (1) and (2). The constant field of $K \cap F$ is $k$ and $K \cap F / k(y)$ is a separable extension, hence $g_{K \cap F} \leq g_{K_{0}}<C$. From Proposition 4.1 we obtain $K \cap F=k(y)$. Given that $F$ is the normal closure of $E_{1} / k(y)$, assertion (2) follows from the Galois correspondence. Let $N$ be the constant field of $E_{1} K$. Since $E_{1} K / K$ is a separable extension, $N / k$ is separable.
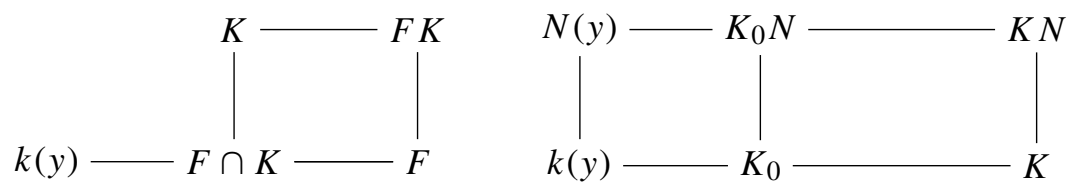

The separable closure of $N(y)$ in $K N$ is $K_{0} N$, hence $F N \cap K N \subseteq K_{0} N$, so $g_{F N \cap K N} \leq g_{K_{0} N}=g_{K_{0}}$. From Proposition 4.1, the genus of each intermediate field $M / N, N(y) \subset M \subseteq F N$, is greater than $C$. Since $N$ is the constant field of $F N \cap K N$, this implies that $F N \cap K N=N(y)$.

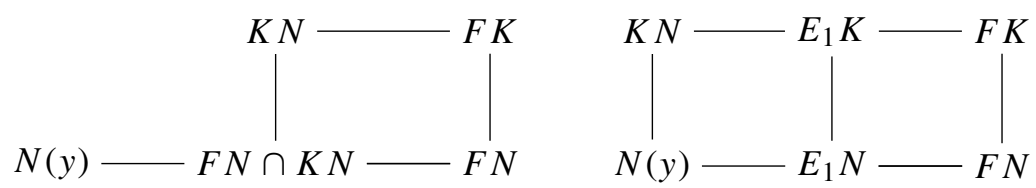

It follows that $\left[E_{1} K: K N\right]=\left[E_{1} N K N: K N\right]=\left[E_{1} N: N(y)\right]$. From (2), we deduce that $\left[E_{1} K: K N\right]=\left[E_{1} K: K\right]$, as $\left[E_{1}: k(y)\right]=\left[E_{1} N: N(y)\right]$. Therefore $K N=K$.

Finally we prove (3). From Proposition 4.1, for every field $E_{2}$ such that $k(y) \subset$ $E_{2} \subseteq E_{1}$, either a place of $k(y)$ of degree greater than $C_{2}$ is ramified or inseparable in $E_{2} / k(y)$, or there are more than $C_{1}$ places of $k(y)$ ramified or inseparable in $E_{2} / k(y)$. Since $C_{1}>n_{s}$ and $C_{2}>d_{s}$, there exists a place of $K_{0}$ of degree greater than $C_{2}$ ramified or inseparable in $E_{2} K_{0} / K_{0}$, or $K_{0}$ has more than $C_{1}-n_{s}$ ramified or inseparable places in $E_{2} K_{0} / K_{0}$.
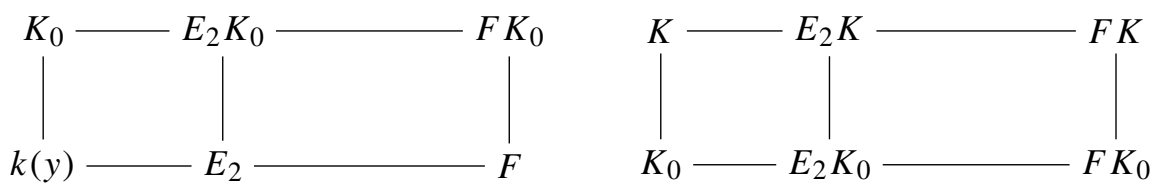

Since $K / K_{0}$ is purely inseparable, by Lemma 2.4 it follows that in $E_{2} K / K$ there exist more than $C_{1}-n_{s}$ ramified or inseparable places, or a place of degree greater than $C_{2}$ is ramified or inseparable. Therefore

$$
\begin{aligned}
g_{E_{2} K} & =1+\left[E_{2} K: K\right]\left(g_{K}-1\right)+\frac{1}{2} \operatorname{deg}\left(D_{E_{2} K / K}\right) \\
& >-m+\frac{1}{2} 2\left(m+C_{0}\right)=C_{0} .
\end{aligned}
$$


We are now in a position to prove the main result of this paper. This is analogous to [8, Satz 1] and [7, Theorem 3].

THEOREM 4.3. Let $E / k$ be a function field with an infinite field of constants and let $E / k(x)$ be a separable extension of degree $m$ such that a place of degree one of $k(x)$ is ramified in $E$. Let $K / k$ be a function field. Then there exist infinitely many nonisomorphic fields $L$ such that $L / K$ is a separable extension of degree $m$ and $\operatorname{Aut}_{K} L=\operatorname{Aut}_{k} L \cong \operatorname{Aut}_{k(x)} E$.

PROOF. Let $\sigma_{1}, \ldots, \sigma_{n-1} \in \operatorname{Aut}_{k} K, \sigma_{i} \neq \operatorname{Id}$ and $\left|\operatorname{Aut}_{k} K\right|=n$. There exist pairwise distinct places $B_{1}, \ldots, B_{n-1}$ of $K$ such that $\sigma_{1}\left(B_{1}\right), \ldots, \sigma_{n-1}\left(B_{n-1}\right)$ are pairwise distinct and $B_{i} \neq \sigma_{j}\left(B_{j}\right)$ with $1 \leq i, j \leq n-1$. By the approximation theorem, there exists $w \in K$ such that $v_{\sigma_{i}\left(B_{i}\right)}(w)>0$ and $v_{B_{i}}(w)=-1$. Denote by $K_{0}$ the separable closure of $k(w)$ in $K$ and by $n_{s}$ the number of places $R$ of $k(w)$ ramified or inseparable in $K_{0} / k(w)$. Choose $d_{s} \in \mathbb{R}$ such that $\operatorname{deg} R \leq d_{s}$.

Let $C_{0}=1+m\left(g_{K}-1\right)+4 m^{2} g_{K}^{2} d$, where $d=\min \left\{\operatorname{deg} B \mid B \in \mathbb{P}_{K}\right\}$. We may assume that the place $Q_{x-1}$ of $k(x)$ is ramified in $E / k(x)$ and that the zero and the pole of $x$ in $k(x)$ are unramified and separable in $E / k(x)$. Consider the function field $F / l$ of Proposition 4.1 with $C>g_{K_{0}}, C_{1}>n_{s}+2\left(m+C_{0}\right), C_{2}>d_{s}, 2\left(m+C_{0}\right)$. From (1) of Proposition 4.1 it follows that for $z=1 / y-1$ the pole $R_{\infty}$ of $z$ is ramified in $F / k(z)$ and its zero $R_{0}$ is unramified and separable. The isomorphism $\varphi: k(w) \rightarrow k(z)$, given by $\varphi(f(w))=f(z)$, can be extended to a homomorphism $\bar{\varphi}$ of $K$ into an algebraic closure $\overline{k(z)}$ of $k(z)$. Therefore we may assume that $K$ is an extension of $k(z)$, and that $K_{0}$ is the separable closure of $k(z)$ in $K$ such that $n_{s}$ is the number of ramified or inseparable places $R$ of $k(z)$ in $K_{0} / k(z)$ and $\operatorname{deg} R \leq d_{s}$. Also may replace $\bar{\varphi}\left(B_{i}\right)$ by $B_{i}$ and $\bar{\varphi} \sigma_{i} \bar{\varphi}^{-1}$ by $\sigma_{i}$ in such a way that $v_{\sigma_{i}\left(B_{i}\right)}(z)>0$ and $v_{B_{i}}(z)=-1$.

Define $L=E_{1} K$. From Proposition 4.2, the field of constants of $L$ is $k$, the degree of $L / K$ is $m$ and $\operatorname{Aut}_{k(z)} E_{1} \cong \operatorname{Aut}_{K} L$. Consider an extension $H_{i} \in \mathbb{P}_{F K}$ of $\sigma_{i}\left(B_{i}\right)$ and the restriction $J_{i}=H_{i} \cap F$.

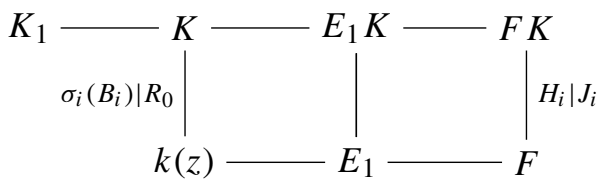

Let $\sigma \in \mathrm{Aut}_{k} L$. From Propositions 3.6 and 4.2, $\sigma(K)=K$. Now, since $R_{\infty}$ is ramified in $F / k(z)$ and the $B_{i}$ are unramified in $K / k(z)$, it follows that each $B_{i}$ is ramified in $F K / K$. Hence, from Lemma 2.3, each $B_{i}$ is ramified or inseparable in $E_{1} K / K$. Since the inertia group $I\left(H_{i} \mid \sigma_{i}\left(B_{i}\right)\right)$ embeds into the inertia group $I\left(J_{i} \mid R_{0}\right)$ and $R_{0}$ is unramified and separable in $F / k(z)$, the places $\sigma\left(B_{1}\right), \ldots, \sigma\left(B_{n-1}\right)$ are unramified and separable in $F K / K$. Then $\sigma \neq \sigma_{i}$. Thus $\operatorname{Aut}_{k} L=\operatorname{Aut}_{K} L$.

Note that since $g_{L}$ can be chosen arbitrarily large, there are infinitely many fields $L$ satisfying the result. If $n=1$ the extension $L$ is obtained as before. 


\section{References}

[1] C. Álvarez-García and G. Villa-Salvador, 'Groups of automorphisms of global function fields', Int. J. Algebra 2 (2008), 65-78.

[2] M. Deuring, Lectures on the Theory of Algebraic Functions of One Variable, Lecture Notes in Mathematics, 314 (Springer, Berlin, 1973).

[3] J. D'Mello and M. Madan, 'Algebraic function fields with solvable automorphism group in characteristic p', Comm. Algebra 11 (1983), 1187-1236.

[4] L. Greenberg, 'Maximal groups and signatures', in: Discontinuous Groups and Riemann Surfaces (Proc. Conf. Univ. Maryland, College Park, MD, 1973) pp. 207-226. Ann. of Math. Studies, No. 79 (Princeton University Press, Princeton, NJ, 1974).

[5] M. Madan and M. Rosen, 'The automorphism group of a function field', Proc. Amer. Math. Soc. 115 (1992), 923-929.

[6] D. J. Madden and R. C. Valentini, 'The group of automorphisms of algebraic function fields', J. Reine Angew. Math. 343 (1983), 162-168.

[7] M. Rzedowski-Calderón and G. Villa-Salvador, 'Automorphisms of congruence function fields', Pacific J. Math. 150 (1991), 167-178.

[8] H. Stichtenoth, 'Zur Realisierbarkeit endlicher Gruppen als Automorphismengruppen algebraischer Funktionenkörper', Math. Z. 187 (1984), 221-225.

[9] H. Stichtenoth, Algebraic Function Fields and Codes, Universitext (Springer, Berlin, 1993).

C. ÁLVAREZ-GARCÍA, Departamento de Control Automático, Centro de Investigación y de Estudios Avanzados del I.P.N.,

Av. Instituto Politécnico Nacional No. 2508,

Col San Pedro Zacatenco, C.P. 07360, México D. F., México

and

Departamento de Matemáticas,

Universidad Autónoma Metropolitana Iztapalapa, México

e-mail: calvarez@ctrl.cinvestav.mx

G. VILLA-SALVADOR, Departamento de Control Automático, Centro de Investigación y de Estudios Avanzados del I.P.N., Av. Instituto Politécnico Nacional No. 2508, Col San Pedro Zacatenco, C.P. 07360, México D. F., México and

Departamento de Matemáticas, Universidad Autónoma Metropolitana Iztapalapa, México

e-mail: gvilla@ctrl.cinvestav.mx 